\title{
Spatial and energy distribution of muons in $\gamma$-induced air showers
}

\author{
A. Fassò \\ Radiation Physics Department, Stanford Linear Accelerator Center, Stanford, CA 94309 \\ J. Poirier \\ University of Notre Dame, Center for Astrophysics, Physics Department, Notre Dame, IN 46556
}

(November 12, 2018)

\begin{abstract}
The FLUKA Monte Carlo program is used to calculate the effects of hadroproduction by primary gamma rays incident upon the earth's atmosphere; for the results presented in this paper, only primary angles at 0 degrees from zenith are considered. The FLUKA code is believed to be quite accurate in reproducing experimental photon hadroproduction data in the $1 \mathrm{GeV}$ to $10 \mathrm{TeV}$ energy range studied. The charged pions which are so produced can decay to muons with sufficient energy to reach ground level. The number of these muons and their radial and energy distribution are studied for incident gamma ray energies from $1 \mathrm{GeV}$ to $10 \mathrm{TeV}$. The number of these muons is not negligible; they can, in certain circumstances, be used to study potential sources of gamma rays like gamma ray bursts. It is found, for example, that a $10 \mathrm{TeV}$ incident primary gamma ray produces, on average, 3.4 muons which reach ground level; the gamma ray energy which produces the maximum number of muons at ground level depends on the spectral index of the primary gamma spectrum, a constant which describes how the primary gamma flux rises with decreasing primary energy. An example: for a differential spectral index of 2.7 , there is a broad maximum number of muons coming from $\approx 30 \mathrm{GeV}$ primary gamma ray energy.
\end{abstract}

PAC numbers: 95.75.Pq,98.70.Sa,98.70.Rz,13.60.Le

\section{INTRODUCTION}

Primary cosmic rays consist mainly of an isotropic flux of charged particles (primarily protons and nuclei). Before reaching the earth, galactic magnetic fields deflect their paths so information about the angular position of their source is lost. On the contrary, neutral particles such as gammas and neutrinos (neutrons decay before reaching the earth) can be directly used to locate the angular position of their origin. Therefore gamma astronomy is important in the study of well localized exotic astrophysical objects. For example, recent reviews are [1.2].

Cosmic gamma rays can be detected directly only by satellite or balloon experiments located essentially outside the earth's atmosphere. The detector size and weight sets limits to the sensitivity and energy range which these experiments can cover which, at present, extends to several tens of $\mathrm{GeV}$. Higher energies are better investigated by means of ground-based experiments which sample the numerous secondary particles produced by the high energy primary photons when they interact in the atmosphere.

Photons constitute only a small fraction of primary cosmic rays. Hadronic showers at ground level are very similar to electromagnetic showers because, at each generation of hadronic pion production, about one-third of the pions are $\pi^{0}$ which immediately decay to gamma rays which then initiate electromagnetic sub-showers. By the time the hadronically initiated shower reaches ground level it is essentially all electromagnetic because of the many generations, each feeding one-third of their energy into the electromagnetic sector. Therefore, gamma showers at ground level are qualitatively similar to those produced by protons and nuclei [3], and experiments based on earth which search for gamma primaries must subtract a large background due to the more numerous cosmic protons and nuclei. Various methods have been developed to discriminate between the two types of showers [1, 2, 4,5 . One of the most commonly used techniques is based on their different muon content [6].

Muons are created in the atmosphere mainly as decay products of charged mesons, which are abundant in hadronic cascades. Pions and kaons can also be produced in the nuclear interactions of high energy primary gamma rays which then initiate secondary hadron showers. The photon cross section for hadronic interactions is about two orders of magnitude smaller than that for producing electron pairs. This low hadronic cross section for gamma rays has been used as a signature for showers initiated by hadrons.

However, muons are present in gamma showers too, albeit in smaller numbers than in a hadronically initiated shower of the same primary energy. The first estimates confirmed the "muon-poor" characteristics of gamma showers [7.8. However, in 1983, Samorski and Stamm [9] reported the results of an extensive air shower experiment at Kiel claiming an excess of events with energies above $2000 \mathrm{TeV}$ centered at the angular location of Cygnus X-3. They also reported a non-deficiency of muons in these data, seemingly inconsistent with a primary gamma ray hypothesis. In 
order to evaluate and discuss these data, many authors performed analytical or Monte Carlo calculations of the muon flux produced in gamma showers 10 21]. Most of these calculations were one-dimensional and all of them referred to gamma energies much larger than $10 \mathrm{TeV}$, appropriate to the Kiel experiment. The energy realm of these calculations was considerably larger than the $1 \mathrm{GeV}$ to $10 \mathrm{TeV}$ region considered in this paper. Therefore, their stress was mostly on sources of muons at very high energies (Bethe-Heitler $\mu$-pair production, decay of charmed particles), with less emphases on muon decay, energy loss, and radial distribution which become important in the energy range considered in this paper.

More recently, the same calculation techniques have been refined and used to optimize current experiments. In addition to an improvement of the adopted cross sections [22], muon energy loss and decay have been added [23, 24], in order to better estimate the sensitivity of modern experiments having a lower muon energy threshold. Several experiments, GRAND [25] and Milagro [26 for example, have the capability of measuring the angles of muons at ground level with high statistics. With the advantage of large numbers of muons, it becomes possible for these experiments to study point sources of gamma rays by studying accumulations of muons at specific angular positions [27 30]. The ability of these experiments to detect a localized source of gamma rays by detecting the secondary muons depends upon several factors such as a) the strength, location, duration, and energy spectrum of the source; b) the angular resolution, detection area and duration of the experiment; and c) the number of muons which reach detection level for the relevant region of primary gamma ray energies due to the physics of the air blanket covering the surface of the earth. A precise calculation of (c) in the $1 \mathrm{GeV}$ to $10 \mathrm{TeV}$ primary gamma ray energy region is the subject of this paper.

A reliable study of muon production by primary gamma rays is necessary in these cases to gauge the sensitivity of the experiments to gamma primaries and to provide information about the expected energy and spatial distribution of muons. Since the threshold energy for detecting the muons has been reduced in these experiments, lower muon energies and lower primary gamma ray energies than previously investigated are studied in this paper. Although the total number of muons per gamma ray at ground level decreases with lower gamma energies, electromagnetic showers produced by photons with lower energies can produce more ground-level muons in total due to the steep increase of gamma flux at lower energies (a spectrum $\mathrm{dN} / \mathrm{dE}=\mathrm{E}^{-\gamma}$, with $\gamma=2.4 \pm 0.4$ [31]) (assuming the primary gamma energy is well above photopion production threshold). Therefore, the new calculations presented here include the primary gamma ray energy range of $1 \mathrm{GeV}<\mathrm{E}_{\gamma}<10 \mathrm{TeV}$ and secondary muon or electron energies above $3 \mathrm{MeV}$. For the results presented in this paper, only primary angles at 0 degrees from zenith are considered.

\section{THE FLUKA PROGRAM}

\section{A. Physics}

FLUKA, unlike most Monte Carlo codes used in cosmic ray research 34 40] is not specialized for this particular field, but is a multipurpose particle transport program with applications as diverse as proton and electron accelerator shielding, calorimetry, medical physics, beam design, high and low energy dosimetry, isotope production, etc. [32, 33]. Recently however, it also has been used successfully in space and cosmic ray studies 41 43].

In FLUKA, different physical models, or event generators, are responsible for the various aspects (particle type, multiplicity, energy and angle) of particle production at different energies. These theoretical models have been directly tested against a large amount of nuclear experimental data, and have also been indirectly validated by comparisons with shower measurements, obtained both at accelerators [44 47] and in cosmic ray experiments [41,48, 49. In particular, FLUKA has been shown to predict hadron-generated muon spectra at different heights in the atmosphere with good accuracy [50].

For the present calculations, the following models are relevant (more details can be found in 450):

- Hadronic interactions above $4 \mathrm{GeV}$ are simulated according to the Dual Parton Model [51]. A list of improvements to the original Monte Carlo version of the model by Ranft [52] can be found in [32].

- A cascade pre-equilibrium model is used for hadronic interactions below $3 \mathrm{GeV}$. The model includes pion and kaon production. Between 3 and $4 \mathrm{GeV}$, inelastic hadron collisions are treated according to a resonance-decay model.

- The Vector Meson Dominance model is used for photonuclear interactions at energies larger than $4 \mathrm{GeV}$. The total cross section is based on experimental photon-proton and photon-neutron cross sections up to and including HERA energies and scaled to photon-nucleus interaction according to Bauer et al. [53]. Shadowing corrections are based on experimental data. The interaction of vector mesons with the nucleus is handled by the Dual Parton Model. 
- Delta Resonance excitation (in the framework of the pre-equilibrium model) is used for photonuclear interactions below $4 \mathrm{GeV}$.

To illustrate the critical role played by event generators in predicting the muon content of showers, the Feynman-x distribution of charged pions (in the lab frame) as calculated by FLUKA is reported in Fig. 1 1 for both gamma and protons at $100 \mathrm{GeV}$. This figure emphasizes a basic difference between gamma and proton-induced showers: the gamma primaries have a larger fraction of high-x secondaries than the proton primaries.

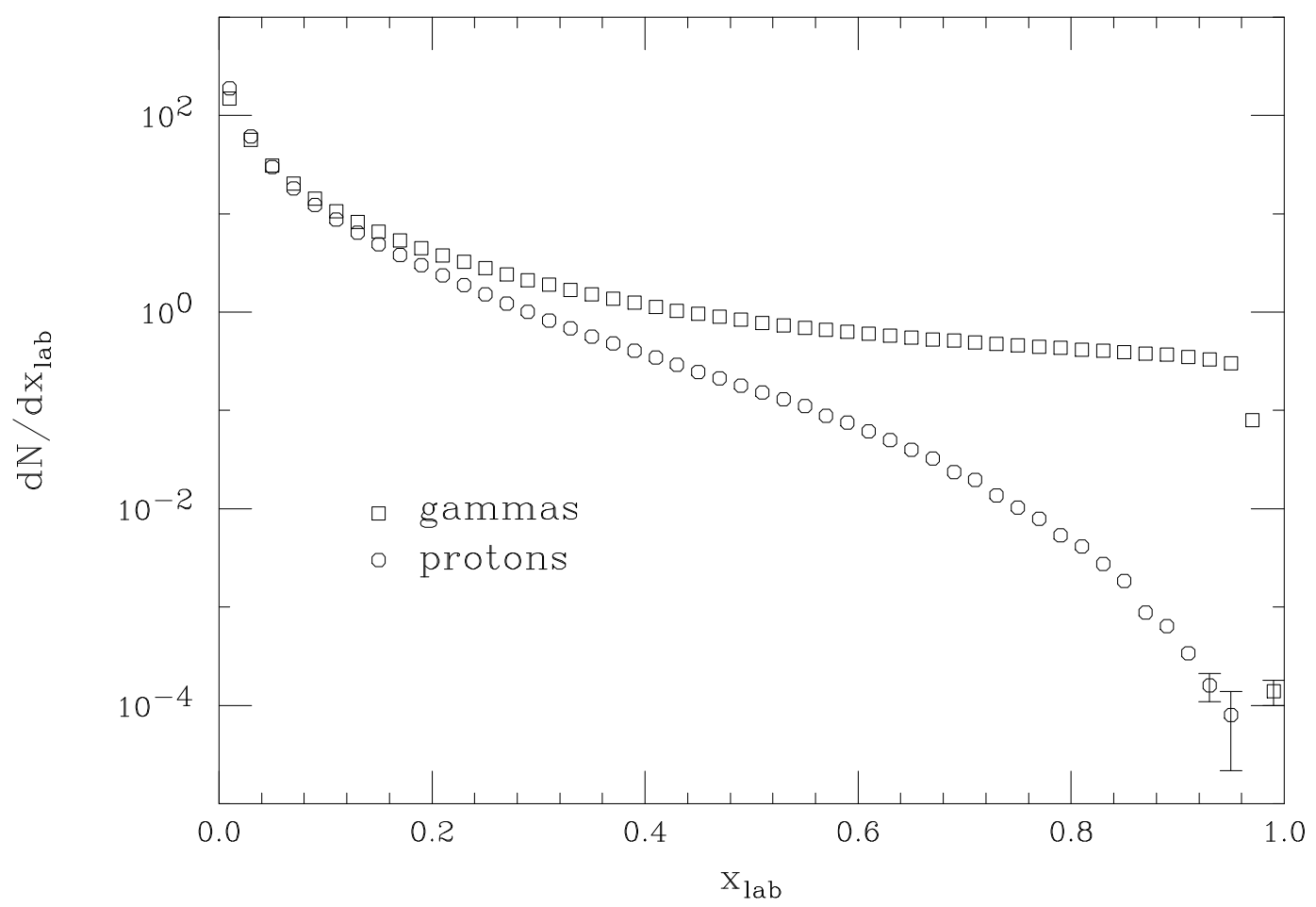

FIG. 1. The Feynman-x distribution of charged pions (in the lab frame) for $100 \mathrm{GeV}$ gamma ray primaries compared with proton primaries of the same energy as contained in the FLUKA Monte Carlo generator for the primary interaction in air.

If we disregard the lower total cross section for gamma rays to produce mesons (about a factor 100), then gammas appear to be more efficient at producing energetic, forward directed pions, and therefore penetrating muons. The gamma primaries give rise to higher energy secondary mesons (on average) and will thus retain a greater fraction of the primary energy in the numerous subsequent hadronic interactions for those which do not decay. Although these differences seem rather substantive, the actual differences which can be observed near sea level may be minor. At any rate, in this paper we only investigate the gamma primaries and leave the comparison with hadronic primaries for future consideration.

The simulation of the electromagnetic cascade in FLUKA is very accurate, including the Landau-PomeranchukMigdal effect and a special treatment of the "tip" of the bremsstrahlung spectrum. Electron pairs and bremsstrahlung are sampled from the proper double differential energy-angular distributions improving the common practice of using average angles. In a similar way, the three-dimensional shape of the hadronic cascades is reproduced in detail by a rigorous sampling of correlated energy and angles in decay, scattering, and multiple Coulomb scattering.

Bethe-Heitler muon pair production is presently being implemented in FLUKA [54,55, but was not yet available at the time of the present calculations; however there is general agreement that this effect is important only for gamma energies greater than several $\mathrm{TeV}$ and for muon energies larger than $1 \mathrm{TeV}$ [10, 14, 16, 18, 22,. Therefore the results presented here should not be affected, with the possible exception of the highest energy point. Charm photoproduction, another possible source of muons, is also not available in FLUKA. According to Berezinskii [56] the corresponding cross section at any energy does not exceed a few percent of other muon producing effects. Each of these effects, if included, would slightly increase the number of muons at ground level above the numbers obtained in this paper. 


\section{B. Variance reduction techniques}

Statistical techniques for accelerating convergence of the results have been used in a few cosmic ray codes, for example MOCCA [39]. In FLUKA, as in most Monte Carlo codes used to solve deep penetration shielding problems, several so-called "biasing" options are available which allow sampling of events having a very small probability. Rigorous proofs of the convergence of results obtained by these techniques to the correct value can be found in specialized books [57,58]. It is important to remember, however, that their use is restricted to the estimation of expectation values and is not appropriate when studying correlations and fluctuations.

In this study, the use of variance reduction techniques has proved essential. It is important to realize that the goal was not to obtain the same results using less computer time, but to include in the study phase space regions which would otherwise not be accessible to Monte Carlo techniques. Due to the very large number of primary photons, some interactions, although extremely rare, may generate events having a finite probability to be detected in an experiment. Below some level of probability per primary photon the computing time required to collect a sufficient number of such events by an unbiased simulation would become prohibitive.

In the present calculations, the following biasing options have been used:

Leading particle biasing: At each electromagnetic interaction with two particles in the final state (bremsstrahlung, pair production, etc.) only one of the two particles is followed, with a probability proportional to its energy. Its statistical weight is modified so as to conserve total weight. This technique, first introduced by A. Van Ginneken [59], is very similar - but not identical - to the so-called "thinning algorithm" of Hillas [39].

Biasing of the mean free path: For interactions having a very small cross section (in our case photonuclear interactions) the cross section is artificially increased by an arbitrary factor chosen by the user (ranging from 10 to 50 in this calculation). The weight of the produced secondaries is reduced so as to conserve probabilities.

Forced decay: A similar technique is used to enhance muon production by artificially decreasing the average decay length of charged mesons. Also in this case, since the weights of both the parent meson and of the produced muon are adjusted by the ratio between the actual and the artificial probability, all the resulting space, energy and angular distributions are correctly reproduced (but with much better statistics in the ranges of interest).

Importance splitting: The loss of statistics due to decrease of particle number with depth in the atmosphere is compensated by replacing a Monte Carlo particle with additional identical particles of lower statistical weight when the particle crosses a boundary between two regions of different pre-defined statistical importance.

\section{CALCULATIONS}

The 1999 version of FLUKA was used to calculate the muon flux and kinetic energy spectrum at various altitudes. At sea level and at $222 \mathrm{~m}$ (the GRAND altitude), muon tracks were scored in 10 concentric rings of radii ranging from $10 \mathrm{~m}$ to $10 \mathrm{~km}$. Photons of energy between $1 \mathrm{GeV}$ and $10 \mathrm{TeV}$ were assumed to enter the atmosphere vertically at $80 \mathrm{~km}$ and the full generated electromagnetic and hadronic showers were followed down to pion production threshold energy. The mean free path for photonuclear interaction was artificially shortened to enhance the sampling frequency by a factor of 10 to 50. As stressed above, the mathematical treatment used ensures that all results are unaffected, while the variances of the average scored quantities (due to the statistical nature of the Monte Carlo technique) are reduced to acceptable levels.

Some approximations were adopted which are expected to have a negligible effect on the results. The atmosphere's geometry was assumed flat and was subdivided into 50 layers for the muon calculation, each of constant density, in order to approximate the exponential character of the earth's air density. Doubling the number of layers from the 25 used in a previous series of calculations did not show any significant difference. The earth's magnetic field was ignored.

For comparison purposes, a similar set of calculations was made to estimate the electron flux at the same positions. All conditions were identical to the muon calculation except the shower energy cutoff was lowered from 150 to $3 \mathrm{MeV}$ to accomodate the lower energies of interest for the electrons and the atmosphere was subdivided into only 25 layers. The word "electron" in this paper is used generically; in all cases it means $\mathrm{e}^{+}+\mathrm{e}^{-}$; as well, "muon" means $\mu^{+}+\mu^{-}$. 


\section{RESULTS}

Fig. 2 2 shows how the average total number of muons and electrons grows with increasing photon energy. A summary of calculated data is reported in Table I. The entries are for gamma ray primaries vertically incident at the top of the earth's atmosphere. All error bars in the figures and tables refer to the statistics of the Monte Carlo calculation.

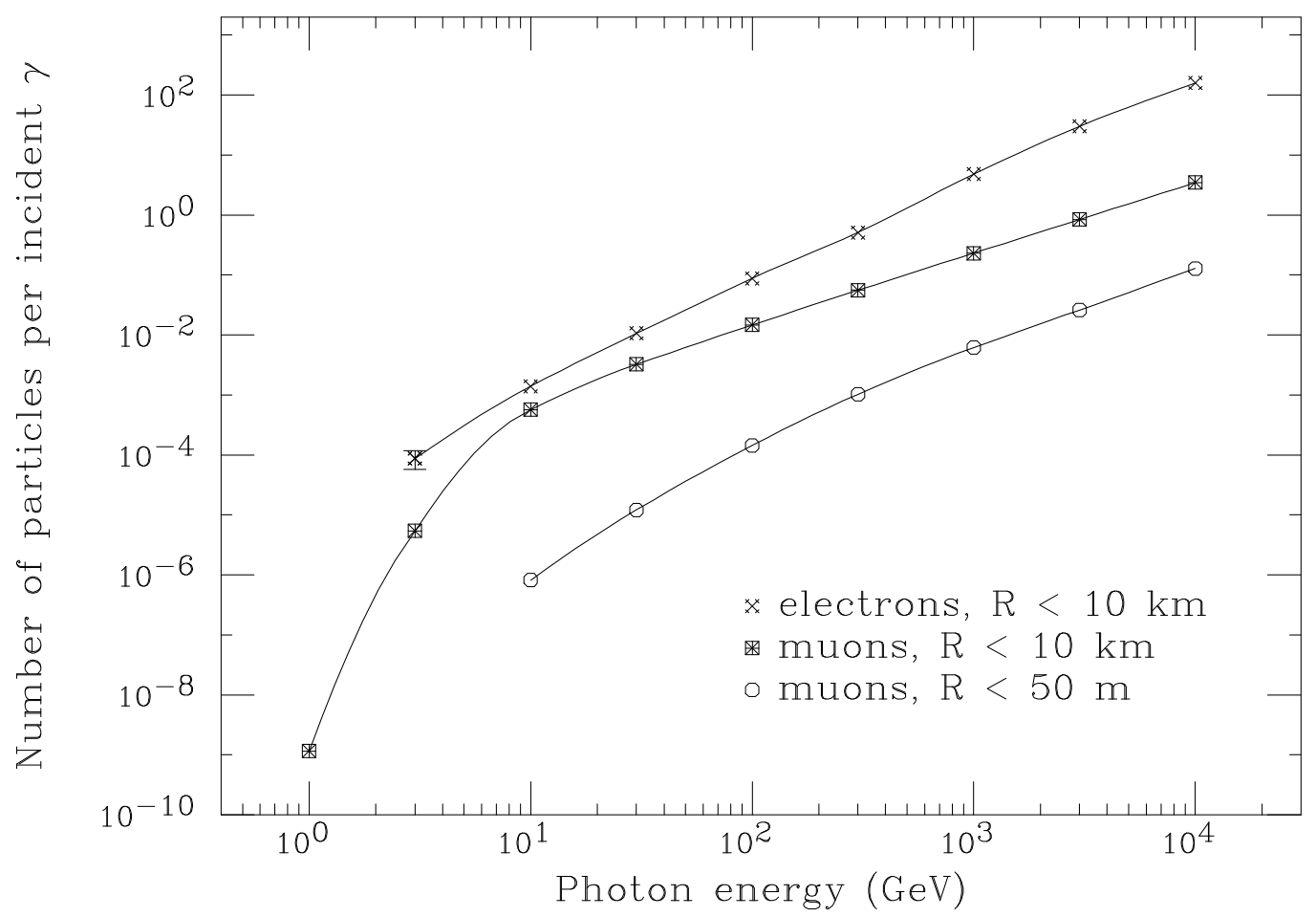

FIG. 2. Number of muons and electrons at $222 \mathrm{~m}$ a.s.l. as a function of incident primary gamma ray energy. The lower points are for secondary muons within a $50 \mathrm{~m}$ radius. 


\begin{tabular}{ccccc}
\hline \hline $\begin{array}{c}\gamma \text { energy } \\
(\mathrm{GeV})\end{array}$ & $222 \mathrm{~m}$ a.s.l. & electrons & & \\
& & sea level & $222 \mathrm{~m}$ a.s.l. & sea level \\
\hline $10^{4}$ & $158 \pm 7$ & $121 \pm 6$ & $3.46 \pm 0.05$ & $3.31 \pm 0.05$ \\
3000 & $30 \pm 3$ & $23 \pm 3$ & $0.840 \pm 0.004$ & $0.807 \pm 0.004$ \\
1000 & $4.8 \pm 0.7$ & $3.7 \pm 0.6$ & $0.2312 \pm 0.0014$ & $0.2230 \pm 0.0014$ \\
300 & $0.51 \pm 0.06$ & $0.41 \pm 0.05$ & $(5.55 \pm 0.08) \times 10^{-2}$ & $(5.37 \pm 0.08) \times 10^{-2}$ \\
100 & $(8.79 \pm 0.06) \times 10^{-2}$ & $(5.84 \pm 0.04) \times 10^{-2}$ & $(1.470 \pm 0.008) \times 10^{-2}$ & $(1.424 \pm 0.008) \times 10^{-2}$ \\
30 & $(1.06 \pm 0.16) \times 10^{-2}$ & $(8.8 \pm 1.4) \times 10^{-3}$ & $(3.25 \pm 0.07) \times 10^{-3}$ & $(3.15 \pm 0.07) \times 10^{-3}$ \\
10 & $(1.4 \pm 0.3) \times 10^{-3}$ & $(1.0 \pm 0.5) \times 10^{-3}$ & $(5.66 \pm 0.11) \times 10^{-4}$ & $(5.45 \pm 0.10) \times 10^{-4}$ \\
3 & $(8.7 \pm 3.0) \times 10^{-5}$ & $(3.8 \pm 1.6) \times 10^{-5}$ & $(5.42 \pm 0.09) \times 10^{-6}$ & $(4.32 \pm 0.09) \times 10^{-6}$ \\
1 & & & $(1.15 \pm 0.19) \times 10^{-9}$ & $(6.9 \pm 1.4) \times 10^{-10}$ \\
\hline \hline
\end{tabular}

TABLE I. Number of secondary muons and electrons per primary gamma ray within a $10 \mathrm{~km}$ radius at 222 and $0 \mathrm{~m}$ above sea level.

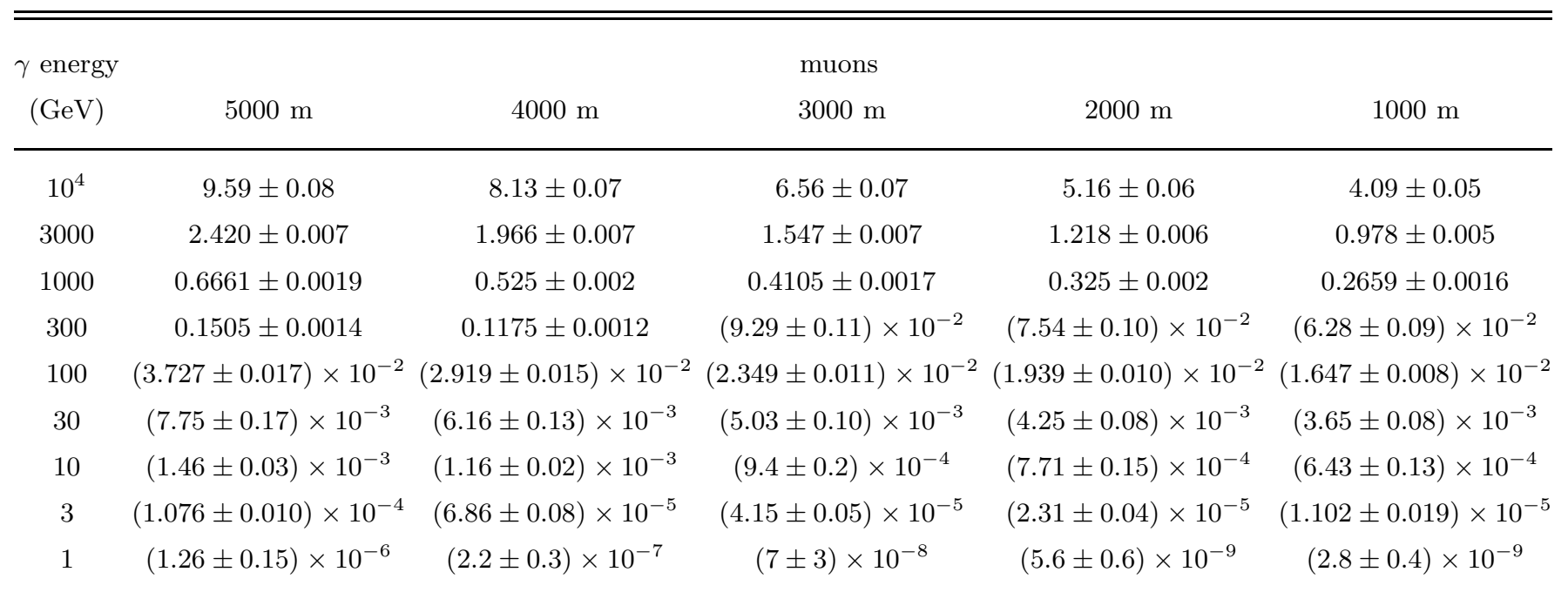

$\overline{\text { TABLE II. Number of secondary muons per incident gamma ray within a } 10 \mathrm{~km} \text { radius at different heights above sea level. }}$ 
Fig. 3 shows how the total number of muons depends upon height above sea level for nine gamma ray primary energies and elevations from 0 to $20 \mathrm{~km}$. The corresponding numerical data are contained in Table II for heights from $1 \mathrm{~km}$ to $5 \mathrm{~km}$. One could interpolate among the values in Table II to estimate the expected number of muons per gamma primary for ground-based detectors at intermediate heights. As expected, the most probable height for each primary energy gradually shifts toward lower heights as the primary energy increases.

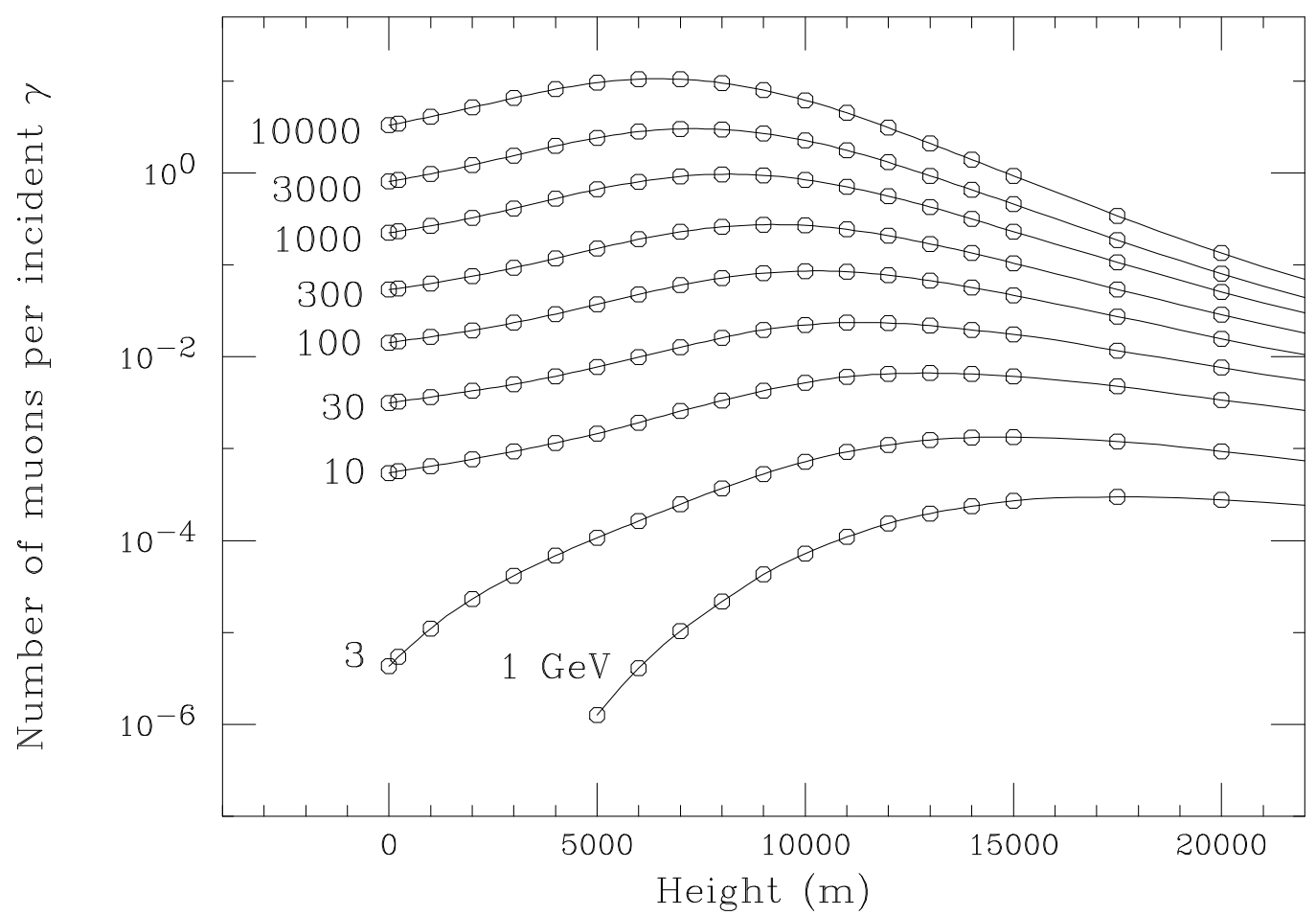

FIG. 3. Average number of secondary muons per incident gamma ray within a $10 \mathrm{~km}$ radius at different heights above sea level. 
The dependence on radial distance is shown in Fig. 1 . The total number of muons within a given radius is plotted as a function of radius. For photon energies $\geq 300 \mathrm{GeV}$, the circle containing half of all the muons reaching sea level has a radius $\leq 700 \mathrm{~m}$, increasing to $\geq 1600 \mathrm{~m}$ for photon energies $\leq 3 \mathrm{GeV}$.

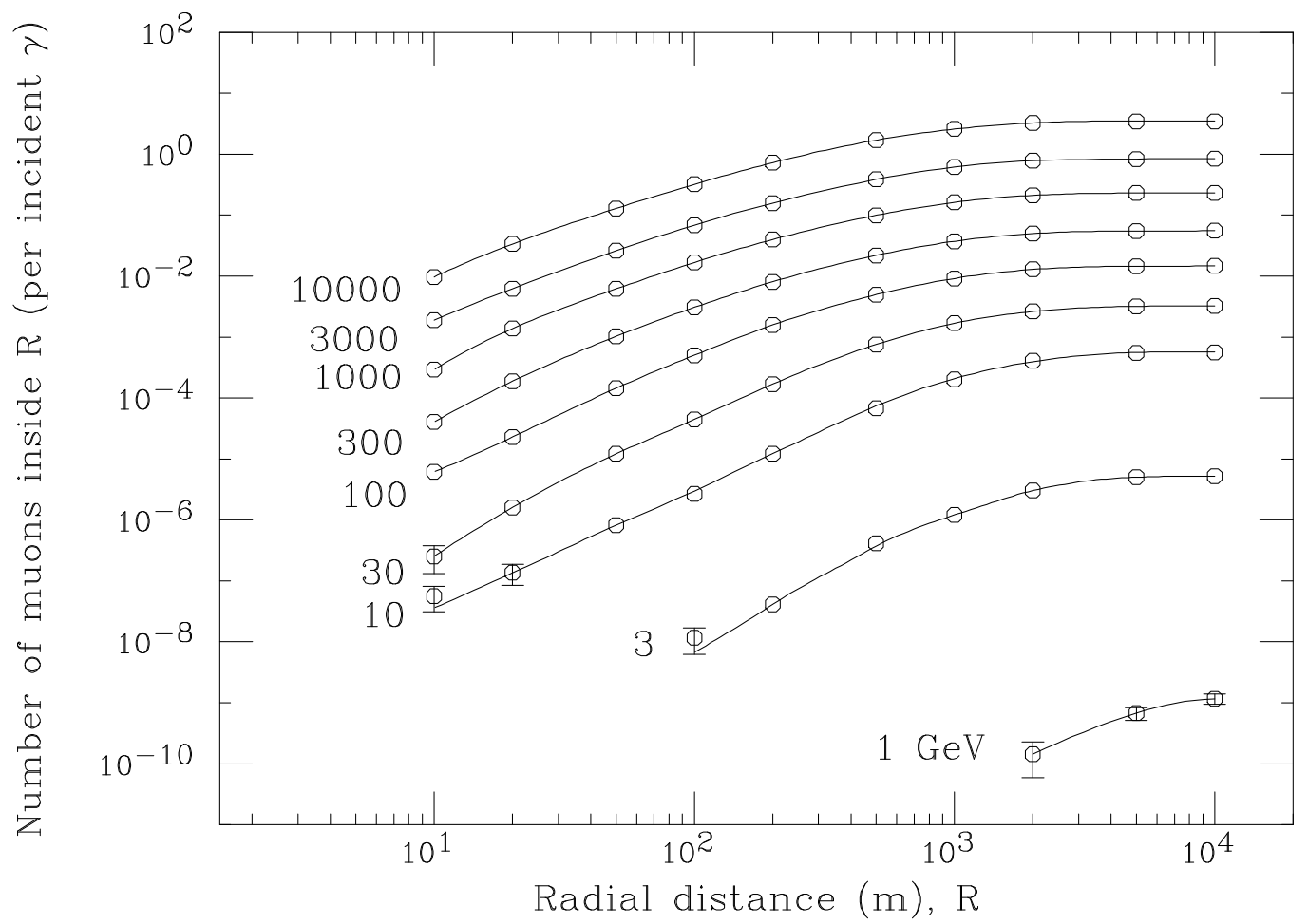

FIG. 4. Number of muons per incident photon within the stated radial distance. Nine incident primary gamma ray energies are shown.

A representative example of the numerous spectra which have been calculated is shown in Fig. 5 which shows nine different primary photon energies from $1 \mathrm{GeV}$ to $10 \mathrm{TeV}$ and gives the muon energy spectra at $222 \mathrm{~m}$ above sea level for those muons at distances $<10 \mathrm{~km}$ (essentially all of them). For a given primary gamma ray energy the points and their error bars are highly correlated due to the summations involved in getting the integrals presented. 


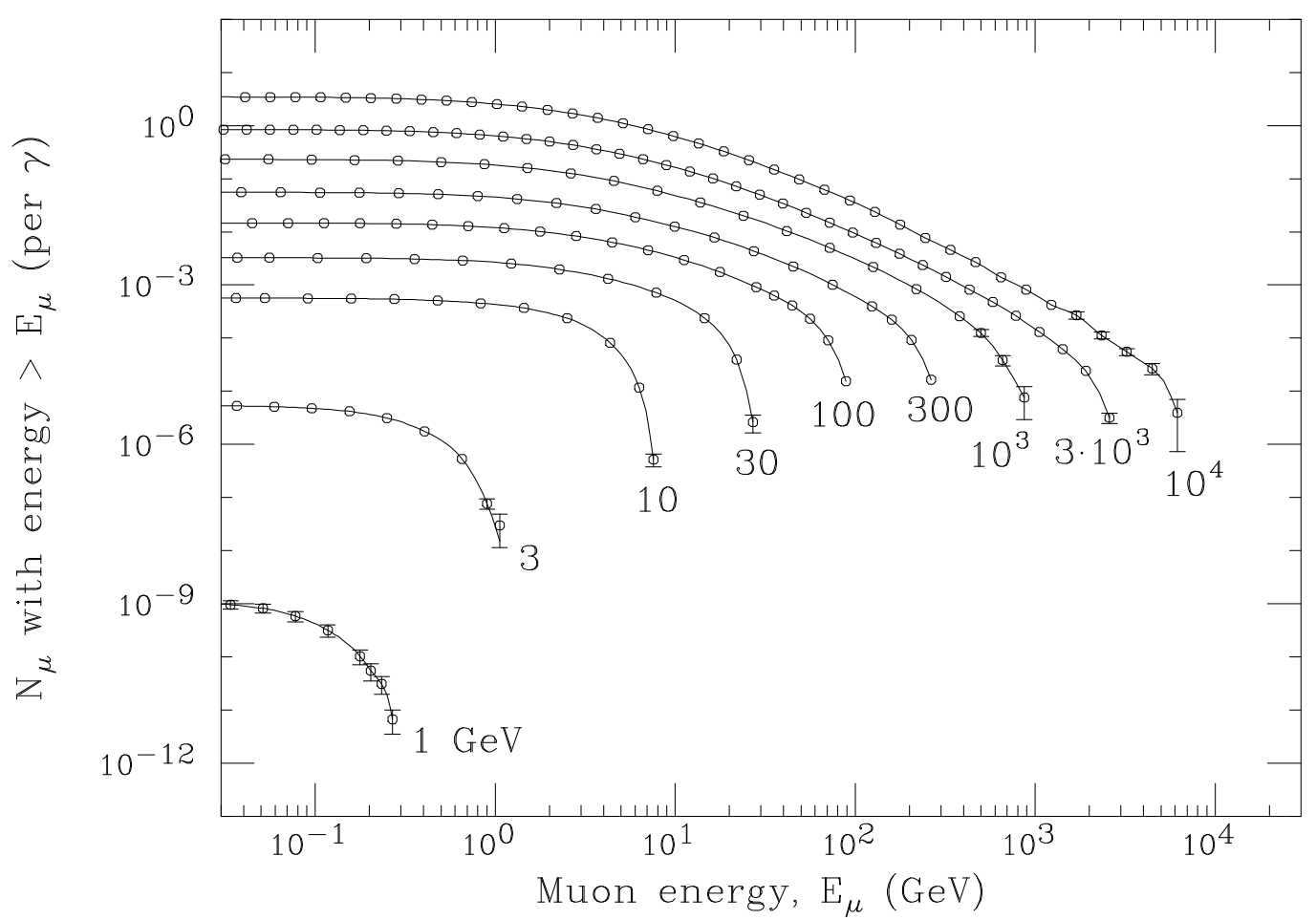

FIG. 5. Integral spectra of muon kinetic energy at radial distances $<10 \mathrm{~km}$ from the shower center, at $222 \mathrm{~m}$ a.s.l.

To estimate the total number of muons reaching detection level, the calculated data should be folded with the incident primary photon spectrum. Assuming a differential energy dependence of $\mathrm{E}^{-2.41}$ [31], it is found that the largest number of muons comes from primary energies of $27 \mathrm{GeV}$. If the spectrum were softer (spectral index, $\gamma=2.77$ ), the corresponding primary energy would drop to $12 \mathrm{GeV}$; if the spectrum were harder $(\gamma=2.05)$, the muon number would continue to increase as the primary energy increases and would depend on where the spectrum softens or cuts off.

\section{CONCLUSIONS}

These reported results are from the Monte Carlo program FLUKA which contains event generators built on the accurate experimental data available in the the energy region of interest. The use of biasing techniques enabled the calculation of events originated by low energy primaries with muon probabilities as low as $\approx 10^{-9}$ per incident gamma ray, which are important since the primary cosmic ray flux rises rapidly with decreasing primary energy. This FLUKA-based Monte Carlo calculation is believed to be quite accurate in this energy region. As such, the results give a good estimate of the number of muons expected from gamma ray primaries in this energy region and help overcome the stigma that muons are only anticoincidence signals for gamma ray primaries. Instead, with the high statistics of the experimental results that are becoming available in this $1 \mathrm{GeV}$ to $10 \mathrm{TeV}$ primary energy region, a statistically significant excess accumulation of muons at a specific angular direction becomes a positive signature for gamma ray initiated hadronic showers and is thus a tool to fill the energy gap between satellite/balloon experiments and Cerenkov arrays. Examples of this use are contained in references [30.60].

Thanks to S. Roesler, M. Dunford, and T. Bowen for their help with this paper. Part of this work was supported by the Department of Energy under contract DE-AC03-76SF00515. Project GRAND is funded through grants from the University of Notre Dame and private donations. 
[1] R.A. Ong, Phys. Rep. 305, 93 (1998).

[2] C.M. Hoffman et al., Rev. Mod. Phys. 71, 897 (1999).

[3] T.K. Gaisser, Cosmic Rays and Particle Physics, Cambridge University Press (1990)

[4] A.M. Hillas, Space Sci. Rev. 75, 17 (1996).

[5] G. Schatz, Space Sci. Rev. 75, 71 (1996).

[6] T.K. Gaisser et al., Phys. Rev. D43, 314 (1991).

[7] S. Karakula and J. Wdowczýk, Acta Phys. Pol. 24, 231 (1963).

[8] T.J.L. McComb, R.J. Protheroe, and K.E. Turver, J. Phys. G5, 1613 (1979).

[9] M. Samorski and W. Stamm, Astrophys. J. 268, L17 (1983).

[10] V.A. Kudryavtsev and O.G. Ryazhskaya, JETP Lett. 42, 371 (1985).

[11] P.G. Edwards, R.J. Protheroe, and E. Rawinski, J. Phys. G11, L101 (1985).

[12] J. Procureur et al., J. Phys. G11, 1377 (1985).

[13] T. Stanev, Ch.P. Vankov and F. Halzen, Univ. of Wisconsin-Madison Report MAD/PH/253, 1985 (unpublished).

[14] T. Stanev and Ch.P. Vankov, Phys. Lett. 158B, 75 (1985).

[15] T. Stanev, T.K. Gaisser, and F. Halzen, Phys. Rev. D32, 1244 (1985)

[16] F. Halzen, K. Hikasa, and T. Stanev, Phys. Rev. D34, 2061 (1986).

[17] T. Stanev, Phys. Rev. D33, 2740 (1986).

[18] V.S. Berezinsky et al., Astron. Astrophys. 189, 306 (1988).

[19] M. Drees, F. Halzen, and K. Hikasa, Phys. Rev. D39, 1310 (1989).

[20] E. Chatelet et al., J. Phys. G16, 317 (1990).

[21] A. Krys, E. Krys, and A. Wasilewski, J. Phys. G17, 1261 (1991).

[22] D.P. Bhattacharyya, Phys. Rev. D55, 2792 (1997).

[23] F. Halzen, T. Stanev, and G.B. Yodh, Phys. Rev. D55, 4475 (1997).

[24] J. Alvarez-Muñiz and F. Halzen, Astrophys. J. 521, 928 (1999).

[25] J. Poirier et al., Preprint astro-ph/0004394 (2000); website: http://www.nd.edu/ grand

[26] Milagro Collaboration, Preprint astro-ph/9912456 (1999).

[27] T.F. Lin et al., Preprint astro-ph/0005180 (2000).

[28] J. Poirier et al., Preprint astro-ph/0004379 (2000).

[29] J.E. McEnery et al., Preprint astro-ph/9910549 (1999).

[30] R. Atkins et al., Preprint astro-ph/0001111 (2000).

[31] R.C. Hartman et al., Astrophys. J. Suppl. Ser. 123, 79 (1999); the average of the differential spectral indices in this Third EGRET Catalog is $2.41 \pm 0.36$.

[32] A. Fassò et al., in Proceedings of the 2nd Workshop on Simulating Accelerator Radiation Environments, CERN 1995, edited by G.R. Stevenson (CERN Report TIS-RP/97-05, 1997), p. 158.

[33] A. Fassò et al., in Proceedings of the 3rd Workshop on Simulating Accelerator Radiation Environments, KEK 1997, edited by H. Hirayama (KEK Proceedings 97-5, 1997), p. 32.

[34] A.K. Konopelko and A.V. Plyasheshnikov, Preprint astro-ph/0001541 (2000).

[35] J. Knapp et al., Karlsruhe Report KfK 6019, 1998 (unpublished).

[36] S.J. Sciutto, Preprint astro-ph/9911331 (1999).

[37] M.P. Kertzman and G.H. Sembroski, Nucl. Instr. Meth. A343, 629 (1994).

[38] V.V. Vassiliev et al., Astropart. Phys. 13, 87 (2000).

[39] A.M. Hillas, Nucl. Phys. B (Proc. Suppl.) 52B, 29 (1997).

[40] C. Forti et al., Phys. Rev. D42, 3668 (1990).

[41] S. Roesler, W. Heinrich, and H. Schraube, Rad. Res. 149, 87 (1998).

[42] G. Battistoni et al., Astropart. Phys. 9, 277 (1998).

[43] G. Battistoni et al., Astropart. Phys. 12, 315 (2000)

[44] A. Fassò et al., Nucl. Instr. Meth. in Phys. Res. A 332, 459 (1993).

[45] A. Ferrari and P.R. Sala, in Proceedings of the Workshop on Nuclear Reaction Data and Nuclear Reactors Physics, Design and Safety, International Centre for Theoretical Physics, Trieste, 1996, edited by A. Gandini and G. Reffo (World Scientific, p. 424 (1998).

[46] A. Ferrari and P.R. Sala, in Proceedings of the International Conference on Nuclear Data for Science and Technology, NDST-97, Trieste, 1997, edited by G. Reffo, A. Ventura, and C. Grandi (Società Italiana di Fisica, Vol. 59, Bologna 1997), Part I, p. 247.

[47] Z. Ajaltouni et al., Nucl. Instr.Meth. in Phys. Res. A387, 333 (1997).

[48] V. Patera et al., Nucl. Instr. Meth. in Phys. Res. A356, 514 (1995).

[49] A. Ferrari, T. Rancati, and P.R. Sala, in Proceedings of the 3rd Workshop on Simulating Accelerator Radiation Environments, KEK 1997, edited by H. Hirayama (KEK Proceedings 97-5, 1997), p. 165.

[50] G. Battistoni et al., Nucl. Phys. B (Proc. Suppl.) 70, 358 (1999).

[51] A. Capella et al., Phys. Rep. 236, 225 (1994).

[52] J. Ranft and S. Ritter, Z. Phys. C20, 347 (1983). 
[53] T.H. Bauer et al., Rev. Mod. Phys. 50, 261 (1978).

[54] G. Baur and A. Leuschner, Eur. Phys. J. C8 631 (1999).

[55] S. Roesler (private communication).

[56] V.S. Berezinskii, V.A. Kudryavtsev, O.G. Ryazskaya, Yad. Fiz. 46, 1716 (1987) [Sov. J. Nucl. Phys. 46, 1026 (1987)].

[57] L.L. Carter and E.D. Cashwell, Particle-Transport Simulation with the Monte Carlo Method (ERDA critical review series, TID-26607, 1975).

[58] I. Lux and L. Koblinger, Monte Carlo Particle Transport Methods: Neutron and Photon Calculations (CRC Press 1991)

[59] A. Van Ginneken, Lecture 14 in Computer Techniques in Radiation Transport and Dosimetry, edited by W.R. Nelson and T.M. Jenkins (Plenum Press, New York 1980), p. 211

[60] J. Poirier et al., Preprint astro-ph/0004379 (2000). 